\title{
Diarrhea as initial manifestation of pulmonary artery intimal sarcoma: a case report and literature review
}

This article was published in the following Dove Press journal:

OncoTargets and Therapy

18 September 2015

Number of times this article has been viewed

Xiaoling Xu'
Ruifeng Zhang'
Huihui Hu'
Wu Ye'
Jin Wang'
Liying Chen'
Lijun Qiu ${ }^{4}$
Kejing Ying'
'Department of Respiratory
Medicine, 'Department of Pathology,
${ }^{3}$ Department of Family Medicine,
${ }^{4}$ Endoscopy Center, Sir Run Run Shaw
Hospital, Medicine School of Zhejiang
University, Hangzhou, People's
Republic of China

Republic of China

\begin{abstract}
Pulmonary artery intimal sarcoma (PAIS) is a rare malignant tumor that presents with nonspecific symptoms and may be misdiagnosed as thromboembolic disease. We report a case of a 40 -year-old female who presented with diarrhea as the initial symptom, was misdiagnosed and received thrombolytic therapy for presumed pulmonary embolism. Progressive symptoms and subsequent surgery led to the diagnosis of PAIS, and early relapse after pulmonary endarterectomy. Her survival time was 17 months after pulmonary endarterectomy. To our knowledge, diarrhea as initial manifestation of PAIS has not been described.
\end{abstract}

Keywords: pulmonary artery intimal sarcoma, chronic diarrhea, soft tissue sarcoma, pulmonary embolism, paraneoplastic syndromes

\section{Introduction}

Pulmonary artery intimal sarcoma (PAIS) is a rare but aggressive malignant mesenchymal tumor, usually arising from the intimal layer of the proximal pulmonary arteries. The usual features of PAIS are intraluminal growth, subsequent vessel obstruction, and proximal or distant metastases. ${ }^{1,2}$ PAIS usually occurs in middle-aged patients with a slight female predominance, and results in high mortality, with a median survival time of approximately 13-18 months. ${ }^{3}$ Since reported by Mandelstamm in 1923, less than 300 cases have been published in the literature, mostly as case reports and case series. Clinical diagnosis of PAIS is often delayed and difficult because of lack of characteristic symptoms. We report here a case of PAIS with diarrhea as initial symptom, misdiagnosed as pulmonary embolism (PE), and early relapse after pulmonary endarterectomy (PEA). To our knowledge, diarrhea as initial manifestation of PAIS has not been described.

\section{Case report}

A 40-year-old female patient was referred to our hospital with complaints of chronic diarrhea and intermittent lower abdominal pain for more than 10 years, and acute exacerbation that had commenced 2 weeks ago. Fecal occult blood test was positive but hemoglobin was normal. Emergency abdominal ultrasonography indicated hepatic congestion, subsequent transthoracic echocardiography demonstrated right ventricular dilation and severe tricuspid valve regurgitation with an estimated right ventricular systolic pressure of $103 \mathrm{mmHg}$ (Figure 1A, B). The N-terminal pro-B-type natriuretic peptide level was 4,654 pg/mL (normal range: 0-125) and D-dimer was $3.28 \mu \mathrm{g} / \mathrm{mL}$ (normal range: 0-0.5). Further evaluation for the cause of pulmonary hypertension was carried out. Computed tomography pulmonary angiogram (CTPA) showed a large filling defect within the main pulmonary trunk and extending into the right and left
Correspondence: Kejing Ying Department of Respiratory Medicine, Sir Run Run Shaw Hospital, No 3 Qing Chun Road East, Hangzhou, Zhejiang 3 I00 I6, People's Republic of China Tel +86 I358870 6900

Fax+86 57I 86044822 Email yingsrrsh@163.com 

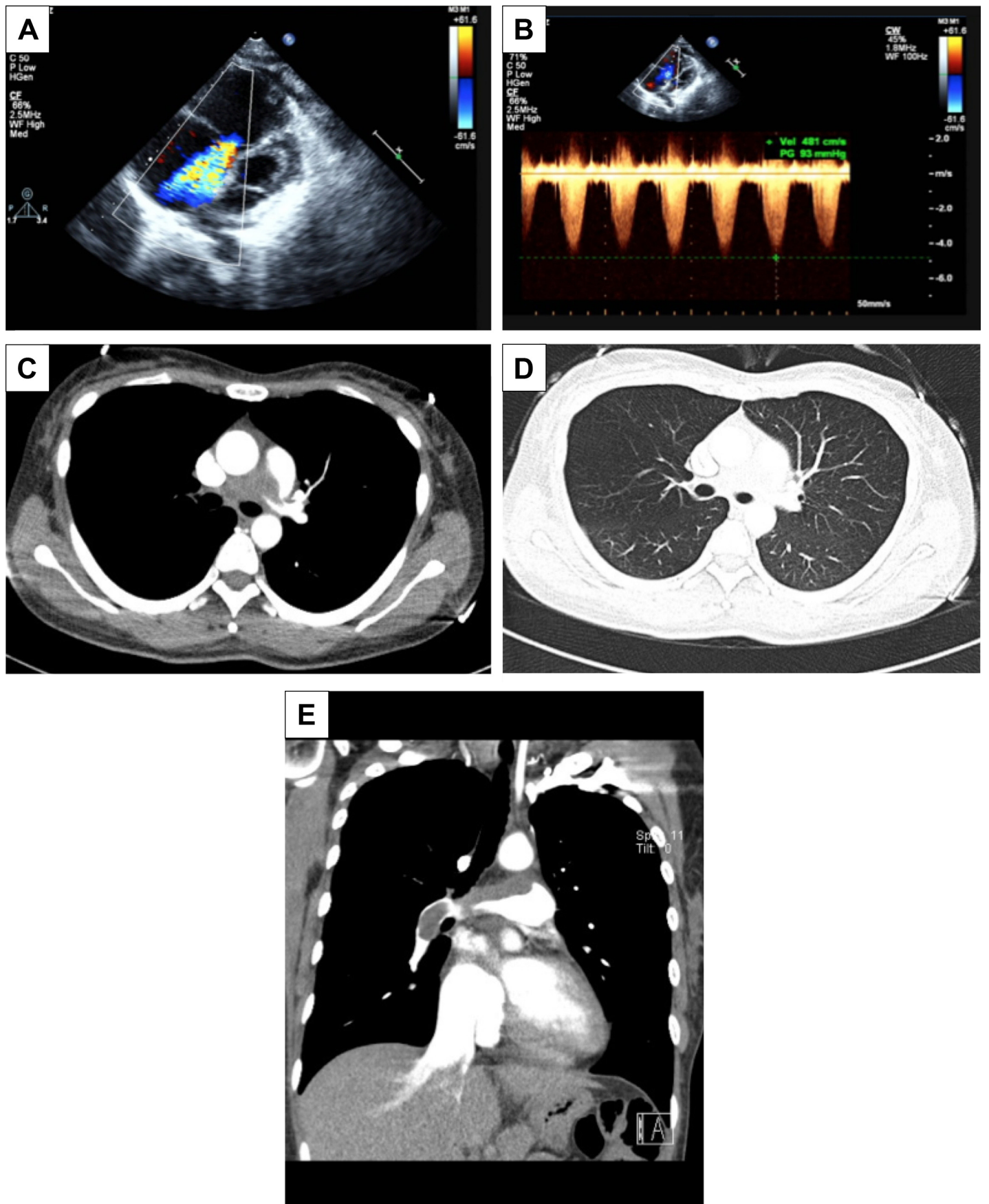

Figure I Echocardiography and CTPA images of the patient.

Notes: (A, B) Echocardiography demonstrated severe tricuspid valve regurgitation with an estimated right ventricular systolic pressure of $103 \mathrm{mmHg}$; (C-E) computed tomography pulmonary angiogram showed filling defect of the pulmonary artery, and there was no tumor in the lung field.

Abbreviation: CTPA, computed tomography pulmonary angiogram.

pulmonary arteries (Figure 1C-E), bilateral lower-extremity venous duplex scan was negative for deep venous thrombosis. She was presumptively diagnosed with acute PE, and intravenous recombinant tissue plasminogen activator (tPA) was administered.

However, 2 days after thrombolytic therapy, she presented with progressive dyspnea and abdominal distension. Ultrasonography indicated moderate pericardial effusion and bilateral pleural effusion. A repeat CTPA revealed no change in size of the pulmonary artery filling defect. Given her history, her presentation with right heart insufficiency, the failure to respond to thrombolytic treatment, and the results of the repeat CTPA, a diagnosis of chronic thromboembolic pulmonary hypertension or malignancy was entertained.

To relieve the patient's symptoms and confirm the diagnosis, an emergency PEA was performed. Macroscopically, 
a whitish-yellow mass obstructed the lumen of the distal main pulmonary trunk, the right pulmonary artery, and the upper left pulmonary artery. Histopathologic examination of the mass showed spindle cells with nuclear pleomorphism, arranged in fascicles and with massive necrosis (Figure 2A). Immunohistochemical staining of the tumor cells was positive for VIM, SMA, Ki-67, and CD99 (Figure 2B-E), but negative for DES (Figure 2F), S100, CK (pan), CD31, CD34, Actin, CD117, and CD68. Based on these findings, a diagnosis of PAIS was made. These findings were in accordance with literature. ${ }^{1,4}$

${ }^{18} \mathrm{~F}$-fluorodeoxyglucose uptake on positron emission computed tomography was performed to confirm the distant metastatic extent of the sarcoma, accumulated radioactivity in middle and lower chest was consistent with changes after surgery, and no other hypermetabolic lesions were observed elsewhere (Figure 3A, B). After surgery, the patient's clinical condition improved and the diarrhea disappeared approximately 1 month later. She was followed-up without further oncological treatments. Six months after surgery, the sarcoma relapsed as an obstructive mass in the right pulmonary artery and the peripheral branches (Figure 3C). Another 5 months later, a right adrenal gland mass was detected by contrastenhanced computed tomography (CT) (Figure 3D) which was pathologically proven to be a metastatic lesion from her sarcoma by puncture biopsy. She underwent $125 \mathrm{I}$ right adrenal gland brachytherapy combined with targeted agent sorafenib since then. Fifteen months after PEA, she was diagnosed with superior vena cava obstruction syndrome, contrast-enhanced CT showed a mass in the right hilum (Figure 3E). The patient suffered symptoms such as chest pain, shortness of breath, and fatigue. No obvious diarrhea during follow-up phase was noticed. Her clinical condition continued to deteriorate and she could not undergo palliative surgery. The patient passed away 17 months after PEA.

\section{Discussion}

Pulmonary artery sarcomas include two types, intimal sarcomas presenting as intraluminal growing excrescences and mural sarcomas involving the pulmonary artery wall, with intraluminal
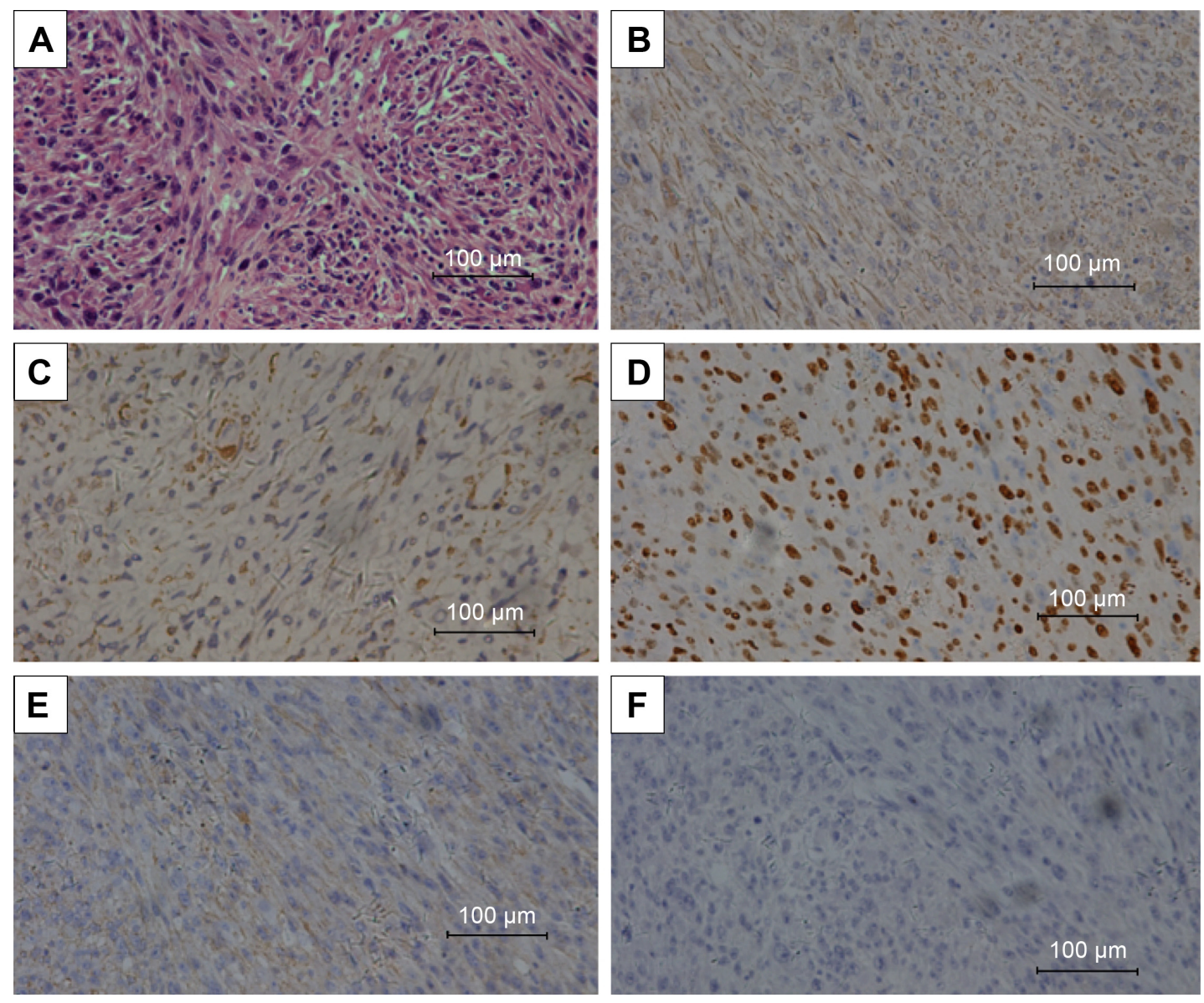

Figure 2 Histopathologic examination and immunohistochemistry staining of tumor cells.

Notes: Histopathologic examination of the tumor showed spindle cells with nuclear pleomorphism, arranged in fascicles and with massive necrosis (A) (hematoxylin and eosin staining; magnification: 200x). Immunohistochemistry staining showed that the tumor cells were positive for VIM (B), SMA (C), Ki-67 (D), and CD99 (E), but negative for DES (F) (magnification: 200x). 

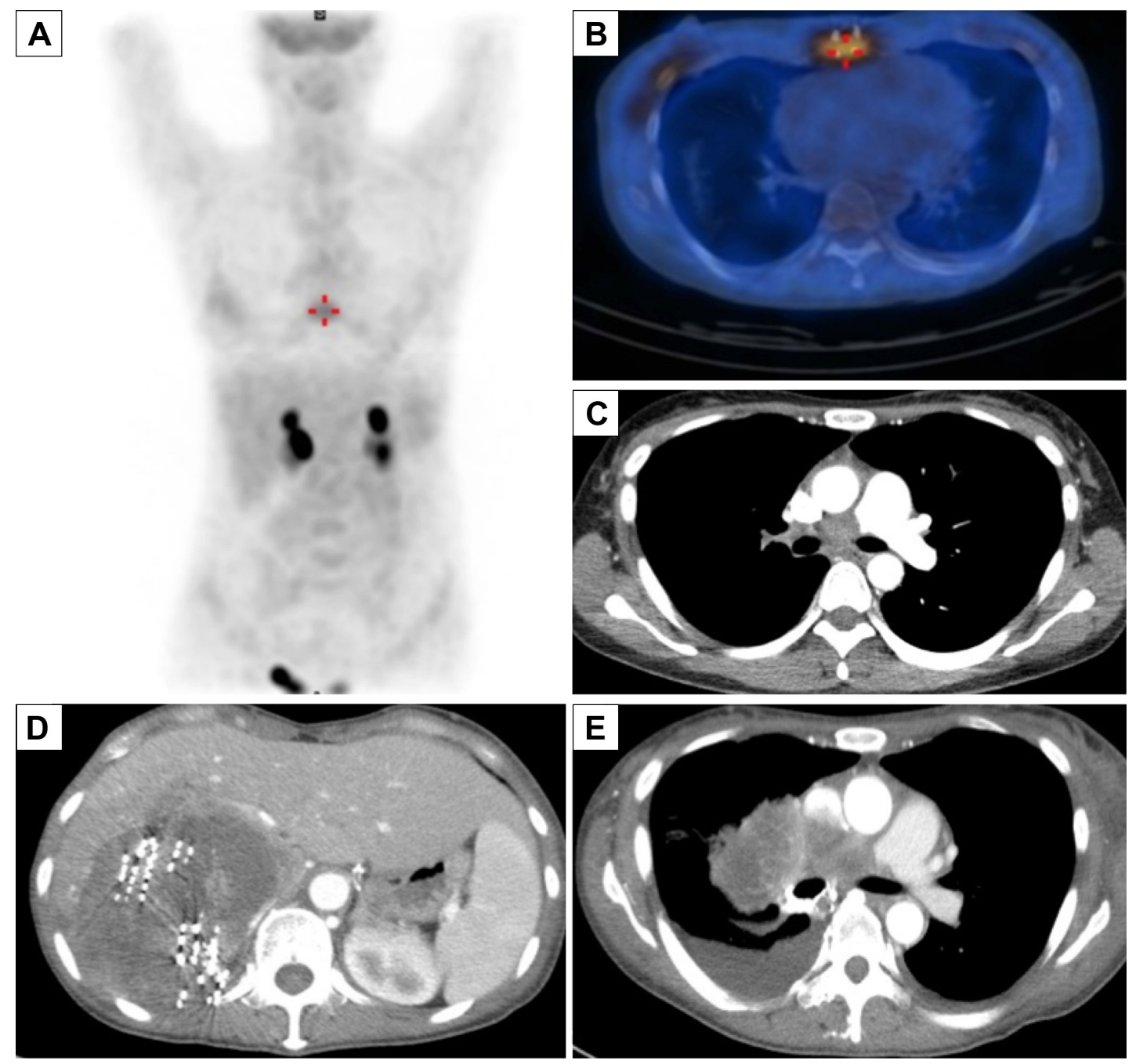

Figure 3 Images of the tumors.

Notes: (A, B) ${ }^{18}$ F-fluorodeoxyglucose uptake on positron emission CT showed accumulated radioactivity in middle and lower chest and no other hypermetabolic lesions were observed elsewhere; (C) CT pulmonary angiogram showed filling defect of the right pulmonary artery; (D) contrast-enhanced CT detected a right adrenal gland tumor after radioactive seed implantation; (E) contrast-enhanced CT detected a mass in the right hilum and pleural effusion.

Abbreviation: CT, computed tomography.

involvement rather than intraparenchymal infiltration. ${ }^{5}$ PAIS arise predominately in the right pulmonary vessels rather than the left. ${ }^{4}$ The definitive diagnosis of PAIS is considered to be difficult and based on pathological examination findings, and specimens are usually obtained from surgery or autopsy. Intimal sarcomas have an unknown cell of origin and usually show undifferentiated cells with variable immunophenotyping. ${ }^{6}$ The true incidence of this malignancy is unknown, and the natural course of this orphan disease remains unclear. Metastases of PAIS occurs commonly; direct infiltration or lung metastases have been reported in $40 \%$ of affected patients while extrathoracic spread has been observed in approximately $16 \%-20 \%$ of cases, ${ }^{1,7}$ involving the lungs, lymph nodes, adrenals, thyroid, brain, liver, pancreas, kidneys, muscles, and skin. Metastatic disease is associated with poor prognosis.
Patients with PAIS generally have a slowly progressive course over weeks to months, ${ }^{8,9}$ manifesting with a variety of cardiopulmonary complaints, atypical symptoms such as dyspnea, chest pain, back pain, cough, hemoptysis, shortness of breath, malaise, or fatigue have been reported. Clinical findings include right ventricular dysfunction, pulmonary hypertension, and pulmonary insufficiency. Multiple gastrointestinal disturbances were typical in patients with advanced cancer, and diarrhea was more common in women than men. ${ }^{10,11}$ In the present case, the initial symptom was diarrhea associated with abdominal pain, presenting as digestive tract congestion. Nevertheless, the digestive discomfort was intermittent for more than 10 years. Her digestive complaints were rare in primary nongastrointestinal stromal tumor soft-tissue sarcomas (STS). Schwenk et al ${ }^{12}$ 
reported a case of primary hemangiosarcoma of the adrenal who presented with diarrhea as initial manifestation. Foale et $\mathrm{al}^{13}$ reported a dog that presented with chronic diarrhea and was diagnosed with left ventricular myosarcoma - symptoms disappeared after surgery. To our knowledge, no study has reported diarrhea as initial manifestation of PAIS before. Paraneoplastic syndromes (PNS) secondary to mesodermal tumors are relatively uncommon. We excluded causes such as gastrointestinal tumor and infectious pathogens. Furthermore, as the patient's gastrointestinal symptoms improved after PEA, we supposed that the patient's chronic diarrhea could be considered as PNS. The advanced tumor caused pulmonary hypertension and right ventricular dysfunction, resulting in digestive tract congestion and presenting as exacerbation of diarrhea. Further studies would be useful to explore the underlying mechanisms of PNS in PAIS.

PAIS often masquerades as other pulmonary vascular diseases because of their similar clinical manifestations and radiologic appearance. At this point, clinicians must pay attention to symptoms that are not commonly associated with pulmonary vascular diseases such as weight loss, anemia, fever, malaise, or fatigue, which are symptoms commonly associated with malignancy. Unheralded or recurrent $\mathrm{PE}$ should also raise the possibility of a malignancy as the underlying cause. Contrast-enhanced CT or CTPA is usually applied as the primary image modality in the differential diagnosis of PAIS. The CT characteristics indicative of sarcoma are unilateral, lobulated, heterogeneously enhancing intravascular masses. Gan et $\mathrm{al}^{14}$ reported that the wall eclipsing sign on contrast-enhanced CT was pathognomonic for PAIS. Positron emission tomography-CT or magnetic resonance imaging is also useful for distinguishing tumors from thrombi. Clinicians ought to consider the possibility of alternative diagnoses as well as PE in patients with pulmonary infarction. The absence of predisposing factors for PE and the lack of response to anticoagulant or thrombolytic therapy are useful for differential diagnoses.

There were no standard therapeutic guidelines and there were few treatment options for the management of intimal sarcoma. PAIS is so rare that it has not been studied in large clinical randomized trials and the results vary in different subtypes of sarcomas. Surgical intervention is known to be the optimal treatment for patients with PAIS, and reported curative surgical procedures for treating the tumor include tumor endarterectomy, graft reconstruction of the pulmonary artery, and pneumonectomy. ${ }^{15}$ An analysis of 20 cases by Wong et al suggested that patients who underwent PEA had significant alleviation of symptoms, even though surgery did not result in a statistically significant survival benefit. ${ }^{16}$ Nonsurgical methods include chemotherapy, radiotherapy, and targeted therapy. Chemotherapeutic agents such as anthracyclines, ifosfamide, gemcitabine with docetaxel, and dacarbazine are recommended in advanced STS, ${ }^{17}$ but at the expense of toxicity and without a clear survival advantage. Radiotherapy, for instance, external beam radiation therapy, has been used to treat residual or unresectable intimal tumors. ${ }^{18}$ The development of targeted drugs such as sorafenib, bevacizumab, and sunitinib has provided opportunities to evaluate new agents in several specific STS subtypes. Sorafenib is a multi-targeted tyrosine kinase inhibitor used in patients with STS and the response rate to this drug was $14 \%$ in a Phase II trial. ${ }^{19}$ Cytotoxic chemotherapy in combination with targeted agents is considered in advanced STS. Sorafenib with dacarbazine was considered to be an active and safety regimen in patients with pretreated STS and a Phase II trial is ongoing in patients affected by selected sarcoma subtypes. ${ }^{20}$ Unfortunately, the role of this adjuvant therapy or combination therapy remains disappointingly controversial, and even with multimodality treatment, the prognosis for PAIS is dismal. ${ }^{8,21}$ Large clinical randomized trials and trials focusing on specific subtypes of sarcomas are expected in the future.

In conclusion, PAIS is a rare malignancy, and clinical manifestation is atypical and easily misdiagnosed. Clinicians must be aware of the presentation and imaging characteristics of this disease.

\section{Acknowledgments}

This study was approved by the ethics committee of Sir Run Run Shaw Hospital, and informed consent was obtained from this patient on admission.

This work was supported by National Nature Science Foundation of China (grant number 81270107); Chinese Medicine Bureau of Zhejiang Province (grant number 2012ZB099); and Medical Science Research Foundation of Zhejiang Province (grant number 2010KYA112).

\section{Disclosure}

The authors report no conflict of interest in this work.

\section{References}

1. Bode-Leniewska B, Komminoth P. Intimal sarcoma. In: Fletcher CDM, Unni KK, Mertens F, editors. World Health Organization Classification of Tumours. Pathology and Genetics of Tumours Soft Tissue and Bone. Lyon: IARC Press; 2002:223-224.

2. Burke A, Virmani R. Tumors of the great vessels. In: Atlas of Tumor Pathology. Tumors of the heart and great vessels. 3rd ed. Washington DC Armed Forces Institute of Pathology; 1996:211-227. 
3. Seelig MH, Klingler PJ, Oldenburg WA, Blackshear JL. Angiosarcoma of the aorta: report of a case and review of the literature. J Vasc Surg. 1998; 28(4):732-737.

4. Burke AP, Virmani R. Sarcomas of the great vessels. A clinicopathologic study. Cancer. 1993;71(5):1761-1773.

5. Bohn OL, de Leon EA, Lezama O, Rios-Luna NP, Sanchez-Sosa S, Llombart-Bosch A. Pulmonary artery sarcoma with angiosarcoma phenotype mimicking pleomorphic malignant fibrous histiocytoma: a case report. Diagn Pathol. 2012;7:154.

6. Sebenik M, Ricci A Jr, DiPasquade B, et al. Undifferentiated intimal sarcoma of large systemic blood vessels: report of 14 cases with immunohistochemical profile and review of literature. Am J Surg Pathol. 2005;29(9): 1184-1193.

7. Manso L, Alvarez E, Quintela M, Cortes-Funes H, Hitt R. Primary pulmonary artery sarcoma: report of three cases and review of the literature. Clin Lung Cancer. 2007;8(4):277-281.

8. Blackmon SH, Rice DC, Correa AM, et al. Management of primary pulmonary artery sarcomas. Ann Thorac Surg. 2009;87(3):977-984.

9. Parish JM, Rosenow EC 3rd, Swensen SJ, Crotty TB. Pulmonary artery sarcoma: clinical features. Chest. 1996;110(6):1480-1488.

10. Komurcu S, Nelson KA, Walsh D, Ford RB, Rybicki LA. Gastrointestinal symptoms among inpatients with advanced cancer. Am J Hosp Palliat Care. 2002;19(5):351-355.

11. Curtis EB, Krech R, Walsh TD. Common symptoms in patients with advanced cancer. J Palliat Care. 1991;7(2):25-29.

12. Schwenk W, Sarbia M, Haas R, et al. Primäres Hämangiosarkom als seltene Form eines Inzidentaloms der Nebenniere. [Primary hemangiosarcoma as a rare form of an incidentally discovered mass of the adrenal glands]. Dtsch Med Wochenschr. 1994;119(7):217-221. German.
13. Foale RD, White RA, Harley R, Herrtage ME. Left ventricular myxosarcoma in a dog. J Small Anim Pract. 2003;44(11):503-507.

14. Gan HL, Zhang JQ, Huang XY, Yu W. The wall eclipsing sign on pulmonary artery computed tomography angiography is pathognomonic for pulmonary artery sarcoma. PLoS One. 2013;8(12):e83200.

15. Tanaka A, Shirasaka T, Okada K, Okita Y. Aggressive multiple surgical interventions to pulmonary artery sarcoma. Eur J Cardiothorac Surg. 2015;47(2):384-385.

16. Wong HH, Gounaris I, McCormack A, et al. Presentation and management of pulmonary artery sarcoma. Clin Sarcoma Res. 2015;5(1):3.

17. von Mehren M, Rankin C, Goldblum JR, et al. Phase 2 Southwest Oncology Group-directed intergroup trial (S0505) of sorafenib in advanced soft tissue sarcomas. Cancer. 2012;118(3):770-776.

18. Choi EY, Yoon YW, Kwon HM, et al. A case of pulmonary artery intimal sarcoma diagnosed with multislice CT scan with 3D reconstruction. Yonsei Med J. 2004;45(3):547-551.

19. Maki RG, D'Adamo DR, Keohan ML, et al. Phase II study of sorafenib in patients with metastatic or recurrent sarcomas. J Clin Oncol. 2009; 27(19):3133-3140.

20. Vincenzi B, Silletta M, Schiavon G, et al. Sorafenib and dacarbazine in soft tissue sarcoma: a single institution experience. Expert Opin Investig Drugs. 2013;22(1):1-7.

21. Mussot S, Ghigna MR, Mercier O, et al. Retrospective institutional study of 31 patients treated for pulmonary artery sarcoma. Eur J Cardiothorac Surg. 2013;43(4):787-793.
OncoTargets and Therapy

\section{Publish your work in this journal}

OncoTargets and Therapy is an international, peer-reviewed, open access journal focusing on the pathological basis of all cancers, potential targets for therapy and treatment protocols employed to improve the management of cancer patients. The journal also focuses on the impact of management programs and new therapeutic agents and protocols on

\section{Dovepress}

patient perspectives such as quality of life, adherence and satisfaction The manuscript management system is completely online and includes a very quick and fair peer-review system, which is all easy to use. Visit http://www.dovepress.com/testimonials.php to read real quotes from published authors. 